\title{
ASYMPTOTIC METHODS IN NUMBER THEORY AND ALGEBRAIC GEOMETRY
}

\author{
by
}

Philippe Lebacque \& Alexey Zykin

\begin{abstract}
The paper is a survey of recent developments in the asymptotic theory of global fields and varieties over them. First, we give a detailed motivated introduction to the asymptotic theory of global fields which is already well shaped as a subject. Second, we treat in a more sketchy way the higher dimensional theory where much less is known and many new research directions are available.

Résumé. - Cet article est un survol des développements récents dans la théorie asymptotique des corps globaux et des variétés algébriques définies sur les corps globaux. Dans un premier temps, nous donnons une introduction détaillée et motivée à la théorie asymptotique des corps globaux, théorie déjà bien établie. Puis nous aborderons plus rapidement la théorie asymptotique en dimension supérieure où peu de choses sont connues et où bien des directions de recherche sont ouvertes.
\end{abstract}

\section{Introduction: the origin of the asymptotic theory of global fields}

The goal of this article is to give a survey of asymptotic methods in number theory and algebraic geometry developed in the last decades. The problems that are treated by the asymptotic theory of global fields (that is number fields or function fields) and varieties over them are quite diverse in nature. However, they are connected by the use of zeta functions, which play the key role in the asymptotic theory.

We begin by a very well known problem which lies at the origin of the asymptotic theory of global fields. Let $\mathbb{F}_{r}$ be the finite field with $r$ elements. For a smooth projective curve $C$ over $\mathbb{F}_{r}$ we let $N_{r}(C)$ be the number of $\mathbb{F}_{r}$-point on $C$. We denote by $g(C)$ be the genus of $C$.

2000 Mathematics Subject Classification. - 11R42,11R29,11G40.

Key words and phrases. — Towers of global fields, L-functions in family, Brauer-Siegel theorem.

The first author was partially supported by EPSRC grant EP/E049109 "Two dimensional adelic analysis". The second author was partially supported by Dmitry Zimin's "Dynasty" foundation, by AG Laboratory SU-HSE, RF government grant, ag. 11.G34.31.0023, by the grants RFBR 10-01-93110-CNRSa, RFBR 11-01-00393-a, by the grant of the Ministry of Education and Science of Russia No. 2010-1.3.1-111-017-029. The individual research project N 10-01-0054 "Asymptotic properties of zeta functions" was carried out with the support of the program "Scientific fund of HSE". 
The problem consists of finding the maximum $N_{r}(g)$ of the numbers $N_{r}(C)$ over all smooth projective curves of genus $g$ over $\mathbb{F}_{r}$ :

$$
N_{r}(g)=\max _{g(C)=g} N_{r}(C)
$$

The first upper bound was discovered by André Weil in 1940s as a direct consequence of his proof of the Riemann hypothesis for curves over finite fields. He showed that $N_{r}(C)$ satisfies the inequality

$$
N_{r}(C) \leq r+1+2 g \sqrt{r} .
$$

Weil bound though extremely useful in many applications is far from being optimal. A dramatic search for the improvements of this bound and for the examples giving lower bounds on $N_{r}(g)$ has begun in 1980s with the discovery of Goppa that curves over finite fields with many points can be used to construct good error-correcting codes. To show how important the developments in this area were it suffices to mention the names of some mathematicians who turned their attention to these questions: J.-P. Serre, V. Drinfeld, Y. Ihara, H. Stark, R. Schoof, M. Tsfasman, S. Vlădut, G. van der Geer, K. Lauter, H. Stichtenoth, A. Garcia, etc. As suggested in [Ser85] by J.-P. Serre the cases when $g$ is small and that when $g$ is large require completely different treatment. That is the latter case which interests us in this article. The first major result in this direction was the following theorem of V. Drinfeld and S. Vlădut [DV]:

Theorem 1.1 (Drinfeld-Vlădut). - For any family of smooth projective curves $\left\{C_{i}\right\}$ over $\mathbb{F}_{r}$ of growing genus we have

$$
\limsup _{i \rightarrow \infty} \frac{N_{r}\left(C_{i}\right)}{g\left(C_{i}\right)} \leq \sqrt{r}-1 .
$$

Moreover, in the case, when $r$ is a square this bound turns out to be optimal. The families of curves, attaining this bound are constructed in many different ways: modular curves, Drinfeld modular curves, explicit iterated constructions, etc. We refer the reader to section 4 for more details. This result, significantly improved and then reinterpreted in terms of limit zeta functions by M. Tsfasman and S. Vlădut, lies at the very base of the asymptotic theory of global fields. We will discuss all this in detail in section 2. It is also possible to extend the Drinfeld-Vlădut inequalities to the case of higher dimensional varieties. This serves as a keystone in the construction of the higher dimensional asymptotic theory (see section 5).

We will now turn our attention to yet another source of development of the asymptotic theory, this time in the case of number fields. Let $K$ be an algebraic number field, that is a finite extension of $\mathbb{Q}$. We denote by $n_{K}=[K: \mathbb{Q}]$ its degree, and by $D_{K}$ its discriminant. An important question (both on its own account and due to its applications in various domains of number theory, arithmetic geometry and theory of sphere packings) is to know the rate of grows of discriminants of number fields. The first bound on $D_{K}$ was obtained by $\mathrm{H}$. Minkowsky using the geometry of numbers. This bound was improved more than half a century later by H. Stark, J.-P. Serre and A. Odlyzko ([Sta74], [Ser75], [Odl76], [Od190]) who used analytic methods involving zeta functions. The bounds they prove are as follows: 
Theorem 1.2 (Odlyzko). — For a family of number fields $\left\{K_{i}\right\}$ we have

$$
\log \left|D_{K_{i}}\right| \geq A \cdot r_{1}\left(K_{i}\right)+2 B \cdot r_{2}\left(K_{i}\right)+o\left(n_{K_{i}}\right),
$$

where $r_{1}\left(K_{i}\right)$ and $r_{2}\left(K_{i}\right)$ are respectively the number of real and complex places of $K_{i}$. Unconditionally, we can take $A=\log (4 \pi)+\gamma+1 \approx 60.8, B=\log (4 \pi)+\gamma \approx 22.3$, and, assuming the generalized Riemann Hypothesis (GRH), one can take, $A=\log (8 \pi)+\gamma+\frac{\pi}{2} \approx 215.3, B=$ $\log (8 \pi)+\gamma \approx 44.7$, where $\gamma=0.577$ is Euler's gamma constant.

The fact that GRH drastically improves the results is omnipresent in the asymptotic theory of global fields. Fortunately, GRH is known for zeta functions of curves over finite fields (Weil bounds) and, more generally, of varieties over finite fields (Deligne's theorem), which allows to have both stronger results and simpler proofs in the case of positive characteristic.

M. Tsfasman and S. Vlădut managed to generalize the above inequalities taking into account the contribution of finite places of the fields. In fact, the restriction of the so-called basic inequality proven by M. Tsfasman and S. Vlădut to infinite primes gives us the inequalities of Odlyzko-Serre. If we restrict the basic inequality to finite places we obtain an analogue of the generalized Drinfeld-Vlădut inequality in the case of number fields. The reader will find more information on this in the next section of the paper.

The last, but not least, problem that led to the development of the asymptotic theory of global fields and varieties over them was the Brauer-Siegel theorem. Let $h_{K}$ denote the class number of a number field $K$ and let $R_{K}$ be its regulator. The classical Brauer-Siegel theorem, proven by Siegel ([Sie]) in the case of quadratic fields and by Brauer ([Bra]) in general describes the behaviour of the product $h_{K} R_{K}$ in families of number fields. The initial motivation for it was a conjecture of Gauss on imaginary quadratic fields, however it has got many important applications elsewhere. The theorem can be stated as follows:

Theorem 1.3 (Brauer-Siegel). - For a family of number fields $\left\{K_{i}\right\}$ we have

$$
\lim _{i \rightarrow \infty} \frac{\log \left(h_{K_{i}} R_{K_{i}}\right)}{\log \sqrt{\left|D_{K_{i}}\right|}}=1
$$

provided the family satisfies two conditions:

(i) $\lim _{i \rightarrow \infty} \frac{n_{K_{i}}}{g_{K_{i}}}=0$;

(ii) either $G R H$ holds, or all the fields $K_{i}$ are normal over $\mathbb{Q}$.

It is possible to remove the first and relax the second conditions of the theorem. The first step towards it was made by Y. Ihara in [Iha83] who considered families of unramified number fields. A complete answer (at least modulo GRH) was given by M. Tsfasman and S. Vlădut in [TV02] who showed how to treat this problem in the framework of the asymptotic theory of number fields, in particular using the concept of limit zeta functions. The corresponding question for curves over finite fields is also of great interest since it describes the asymptotic behaviour of the number of rational points on Jacobians of curves over finite fields. All this will be discussed in detail in the section 3 . 
In our introduction we mostly considered the one dimensional case of number fields or function fields. Here the theory is best developed. However, there is quite a number of results and conjectures for higher dimensional varieties with particularly nice arithmetical applications. Some of the results in this actively developing area are discussed in section 5 .

Let us finally say that, despite of the fact that the theory of error correcting codes and the theory of sphere packings are just briefly mentioned in our introduction their role in the creation of the asymptotic theory of global fields is fundamental. Indeed many questions some of which were mentioned here (maximal number of points on curves, growth of the discriminants, etc.) received particular attention due to their relation to error-correcting codes or sphere packings.

\section{Basic concepts and results. Tsfasman-Vlăduţ invariants of infinite global fields}

Many authors considered the behaviour of arithmetic data (decomposition of primes, genus, root discriminant, class number, regulator etc.) in families of global fields. Tsfasman and Vlădut laid the foundation for the asymptotic theory of global fields in order not to consider fields in a family, but the limit object (say, a limit zeta-function) that would encode the information concerning the asymptotics of the initial arithmetic data.

In this section we introduce some definitions and give basic properties of families of global fields.

2.1. Tsfasman-Vlădut invariants. - Arguments and proofs for the results from this subsection can be found in [TV02]. Let us first define the objects we are to work with. Let $r$ be a power of a prime $p$, and let $\overline{\mathbb{F}}_{r}$ denote the algebraic closure of $\mathbb{F}_{r}$.

Definition 2.1. - A family of global fields is a sequence $\mathcal{K}=\left\{K_{n}\right\}_{n \in \mathbb{N}}$ such that:

1. Either all the $K_{n}$ are finite extensions of $\mathbb{Q}$ or all the $K_{n}$ are finite extensions of $\mathbb{F}_{r}(t)$ with $\overline{\mathbb{F}}_{r} \cap K_{n}=\mathbb{F}_{r}$.

2. if $i \neq j, K_{i}$ is not isomorphic to $K_{j}$.

A tower of global fields is a family satisfying in addition $K_{n} \subset K_{n+1}$ for every $n \in \mathbb{N}$. An infinite global (resp. number, resp. function) field is the limit of a tower of global (resp. number, resp. function) fields, i.e. it is the union $\bigcup_{n=1}^{\infty} K_{n}$.

Definition 2.2. - The genus $g_{K}$ of a function field is the genus of the corresponding smooth projective curve. We define the genus of a number field $K$ as $g_{K}=\log \sqrt{\left|D_{K}\right|}$, where $D_{K}$ is the discriminant of $K$.

As there are (up to an isomorphism) only finitely many global fields with genus smaller than a fixed real number $g$, we have the following proposition.

Proposition 2.3. - For any family $\left\{K_{i}\right\}$ of global fields the genus $g_{K_{i}} \rightarrow+\infty$. 
Thus, in the number fields case, any infinite algebraic extension of $\mathbb{Q}$ is an infinite number field, whereas in the function fields case, we require the infinite algebraic extension of $\mathbb{F}_{r}(t)$ to contain a sequence of function fields with genus going to infinity.

Let us now define the so-called Tsfasman-Vlădut invariants of a family of global fields. Throughout the paper, we use the acronyms NF and FF for the number field and the function field cases respectively. As before, the GRH indication means that we assume the generalized Riemann Hypothesis for Dedekind zeta-functions.

First we introduce some notation to be used throughout the paper:

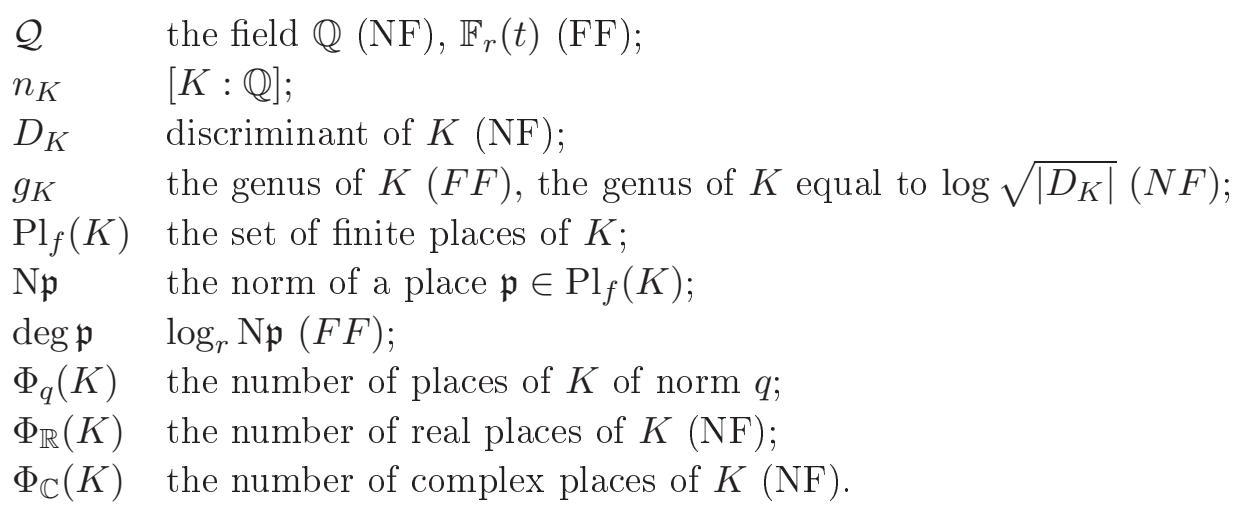

We consider the set of possible indices for the $\Phi_{q}$,

$$
A= \begin{cases}\left\{\mathbb{R}, \mathbb{C}, p^{k} \mid p \text { prime }, k \in \mathbb{Z}_{>0}\right\} & (N F) \\ \left\{r^{k} \mid k \in \mathbb{Z}_{>0}\right\} & (F F)\end{cases}
$$

and $A_{f}$ its subset of finite parameters $\left\{p^{k} \mid p\right.$ prime, $\left.k \in \mathbb{Z}_{>0}\right\}$.

Definition 2.4. - We say that a family $\mathcal{K}=\left\{K_{i}\right\}$ of global fields is asymptotically exact if the following limit exists for any $q \in A$ :

$$
\phi_{q}:=\lim _{i \rightarrow+\infty} \frac{\Phi_{q}\left(K_{i}\right)}{g_{K_{i}}} .
$$

It is said to be asymptotically good if in addition one of the $\phi_{q}$ is nonzero, and asymptotically bad otherwise. The numbers $\phi_{q}$ are called the Tsfasman-Vlădut invariants of the family $\mathcal{K}$.

This definition has two origins. The first one is the information theory since the families giving good algebraic geometric codes are those for which $\phi_{r}$ exists and is big. The second one is more technical and can be seen through Weil's explicit formulae. For convenience we also put $\phi_{\infty}=\lim \frac{n_{K_{i}}}{g_{K_{i}}}=\phi_{\mathbb{R}}+2 \phi_{\mathbb{C}}$.

Being asymptotically exact is not a restrictive condition. To be precise:

Proposition 2.5. — 1. Any family of global fields contains an asymptotically exact subfamily.

2. Any tower of global fields is asymptotically exact and the $\phi_{q}$ 's depend only on the limit. 
We can thus define the Tsfasman-Vlădut invariants of an infinite global fields $\mathcal{K}$ as the invariants of any tower having limit $\mathcal{K}$. From now on, we only consider asymptotically exact families, since they provide natural framework for asymptotic considerations. One of the problems of the asymptotic theory is to understand the set of possible $\left\{\phi_{q}\right\}$. In the next propositions we describe some the general properties of the $\left\{\phi_{q}\right\}$. Let us start with the basic inequalities:

Theorem 2.6 (Tsfasman-Vlădut). - For any asymptotically exact family of global fields, the following inequalities hold:

$$
\begin{aligned}
&(N F-G R H) \sum_{q} \frac{\phi_{q} \log q}{\sqrt{q}-1}+\left(\log \sqrt{8 \pi}+\frac{\pi}{4}+\frac{\gamma}{2}\right) \phi_{\mathbb{R}}+(\log 8 \pi+\gamma) \phi_{\mathbb{C}} \leq 1, \\
&(N F) \quad \sum_{q} \frac{\phi_{q} \log q}{q-1}+\left(\log 2 \sqrt{\pi}+\frac{\gamma}{2}\right) \phi_{\mathbb{R}}+(\log 2 \pi+\gamma) \phi_{\mathbb{C}} \leq 1, \\
&(F F) \quad \sum_{m=1}^{\infty} \frac{m \phi_{r} m}{r^{\frac{m}{2}}-1} \leq 1,
\end{aligned}
$$

where $\gamma$ is the Euler constant.

This result is central in what follows. For instance, it is used to show the convergence of the limit zeta-function associated to the family. It is proven using the Weil explicit formulae, the effective Chebotarev density theorem for number fields and the Riemann hypothesis for function fields.

In the case of towers of number fields (and of function fields if we consider suitable quantities), the degree of the extension gives an upper bound for the number of places above a prime number $p$ :

Proposition 2.7. - For an asymptotically exact family of number fields and any prime number $p$ the following inequality holds:

$$
\sum_{m=1}^{+\infty} m \phi_{p^{m}} \leq \phi_{\mathbb{R}}+2 \phi_{\mathbb{C}}
$$

Let us finally define the deficiency $\delta_{\mathcal{K}}$ of an asymptotically exact family $\mathcal{K}=\left\{K_{i}\right\}$ of global fields as the difference between the two sides of the basic inequalities under GRH:

$$
(N F) \quad \delta_{\mathcal{K}}=1-\sum_{q} \frac{\phi_{q} \log q}{\sqrt{q}-1}-\left(\log \sqrt{8 \pi}+\frac{\pi}{4}+\frac{\gamma}{2}\right) \phi_{\mathbb{R}}-(\log 8 \pi+\gamma) \phi_{\mathbb{C}}
$$

and

$$
(F F) \quad \delta_{\mathcal{K}}=1-\sum_{m=1}^{\infty} \frac{m \phi_{r^{m}}}{r^{\frac{m}{2}}-1} .
$$

A remarkable fact is that the deficiency of infinite global fields is increasing with respect to the inclusion (see [Leb10]): $\mathcal{K} \subset \mathcal{L}$ implies $\delta_{\mathcal{K}} \leq \delta_{\mathcal{L}}$. One knows that fields of zero deficiency exist in the function fields case (c.f. section 4). Such infinite global fields are called optimal, and they are of particular interest for the information theory. 
2.2. Ramification, prime decomposition and invariants. - The precise statements and proofs of the results from this subsection can be found in [GSR] and [Leb10]. The Tsfasman-Vlădut invariants of infinite global fields contain information on the ramification and the decomposition of places in these fields. Indeed, one sees from Hurwitz genus formula that any finitely ramified and tamely ramified tower of number fields is asymptotically good (because it has bounded root discriminant). For function fields, we have to ask in addition for the existence of a split place. It is not excluded that there exists an asymptotically good infinite global field with infinitely many ramified places and no split place, but no examples have been found so far. In the case of function fields, A. Garcia and H. Stichtenoth provided a widely ramified optimal tower and an everywhere ramified tower of function fields with bounded $g / n$ is constructed in [DPZ]. Unfortunately, we do not know anything similar for number fields.

In general, we expect asymptotically good towers to have very little ramification and some split places. The next question, first raised by Y. Ihara, is how many places split completely in a tower $\mathcal{K}$ of global field. It follows from the Chebotarev density theorem that the set of completely split places has in general a zero analytic density, that is

$$
\lim _{s \rightarrow 1^{+}} \frac{\sum_{\mathfrak{p} \in D} \mathrm{~Np}^{-s}}{\sum_{\mathfrak{p} \in \mathrm{Pl}_{f}(\mathcal{Q})} \mathrm{Np}^{-s}}=0,
$$

where $D$ is the set of places of $\mathcal{Q}$ that split completely in $\mathcal{K} / \mathcal{Q}$. In the case of asymptotically good fields, $\sum_{\mathfrak{p} \in D} \mathrm{~Np}^{-1}$ is even bounded. However, in the case of asymptotically bad fields, the numerator can have an infinite limit whereas the ramification locus is very small (but infinite). We refer the reader to [Leb10] for a more detailed treatment of the above questions.

\section{Generalized Brauer-Siegel theorem and limit zeta-functions}

3.1. Generalizations of the Brauer-Siegel theorem. - Now we turn our attention to the Brauer-Siegel theorem. The in-depth study of mathematical tools involved in it leads to an important notion of limit zeta functions which plays a key role in the study of asymptotic problems.

While looking at the statement of the Brauer-Siegel theorem (theorem 1.3) one immediately asks a question whether the two conditions present in it are indeed necessary. It is a right guess that the second condition involving normality is technical in its nature (though getting rid of it would be a breakthrough in the analytic number theory since it is related to the so-called Siegel zeroes of zeta-functions - the real zeroes which lie abnormally close to $s=1$; of course, presumably they do not exist). The second condition $n_{K} / \log \sqrt{\left|D_{K}\right|} \rightarrow 0$ looks much trickier. Using the inequalities from proposition 2.7 it is immediate that this condition is equivalent to the fact that the family we consider is asymptotically bad.

A fundamental theorem of M. Tsfasman and S. Vlădut from [TV02] allows both to treat the asymptotically good case of the Brauer-Siegel theorem and to relax the second condition. 
We formulate it together with a complementary result by A. Zykin [Zyk05] which relaxes the second condition in the asymptotically bad case. Before stating the result we give the following definition:

Definition 3.1. - We say that a number field $K$ is almost normal if there exists a tower $K=K_{n} \supset \cdots \supset K_{1} \supset K_{0}=\mathbb{Q}$, where each step $K_{i} / K_{i-1}$ is normal.

Theorem 3.2 (Tsfasman-Vlădut-Zykin). - Assume that for an asymptotically exact family of number fields $\left\{K_{i}\right\}$ either GRH holds or all the fields $K_{i}$ are almost normal. Then we have:

$$
\lim _{i \rightarrow \infty} \frac{\log \left(h_{K_{i}} R_{K_{i}}\right)}{g_{K_{i}}}=1+\sum_{q} \phi_{q} \log \frac{q}{q-1}-\phi_{\mathbb{R}} \log 2-\phi_{\mathbb{C}} \log 2 \pi,
$$

the sum being taken over all prime powers $q$.

For an asymptotically bad family of number fields we have $\phi_{\mathbb{R}}=0$ and $\phi_{\mathbb{C}}=0$ as well as $\phi_{q}=0$ for all prime powers $q$, so the conclusion of the theorem takes the form of that of the classical Brauer-Siegel theorem. However, there are examples of families of number fields where the right hand side of the equality in the theorem is either strictly less or strictly greater than one (see [TV02]). Let us mention one particularly nice corollary of the generalized BrauerSiegel theorem due to M. Tsfasman and S. Vlăduţ: a bound on the regulators that improves Zimmert's bound (see [Zim], his bound can be written as $\lim \inf \frac{\log R_{K_{i}}}{g_{K_{i}}} \geq(\log 2+\gamma) \phi_{\mathbb{R}}+$ $\left.2 \gamma \phi_{\mathbb{C}}\right)$.

Theorem 3.3 (Tsfasman-Vlădut). - For a family of almost normal number fields $\left\{K_{i}\right\}$ (or any number fields under the assumption of GRH) we have

$$
\liminf \frac{\log R_{K_{i}}}{g_{K_{i}}} \geq(\log \sqrt{\pi e}+\gamma / 2) \phi_{\mathbb{R}}+(\log 2+\gamma) \phi_{\mathbb{C}}
$$

The proof of this bound is far from being trivial, it can be found in [TV02].

The function field version of the Brauer-Siegel theorem is both easier to prove and requires no supplementary conditions (like normality or GRH). In fact, it was obtained before the corresponding theorem for number fields and allowed to guess what the result for number fields should be (for a proof see [Tsf92] or [TV97]).

Theorem 3.4 (Tsfasman-Vlădut). - For an asymptotically exact family of smooth projective curves $\left\{X_{i}\right\}$ over a finite field $\mathbb{F}_{r}$ we have:

$$
\lim _{i \rightarrow \infty} \frac{\log h_{i}}{g_{i}}=\log r+\sum_{f=1}^{\infty} \phi_{r^{f}} \log \frac{r^{f}}{r^{f}-1}
$$

where $h_{i}=h\left(X_{i}\right)=\left|\left(\operatorname{Jac} X_{i}\right)\left(\mathbb{F}_{r}\right)\right|$ is the cardinality of the Jacobian of $X_{i}$ over $\mathbb{F}_{r}$. 
Let $\varkappa_{K}=\operatorname{Res}_{s=1} \zeta_{K}(s)$ be the residue of the Dedekind zeta function $\zeta_{K}(s)=\prod_{q}\left(1-q^{-s}\right)^{-\Phi_{q}(K)}$ of the field $K$ at $s=1$. Using the residue formula (see [Lan94, Chapter VIII] and [TVN, Chapter III])

$$
\begin{gathered}
\varkappa_{K}=\frac{2^{\Phi_{\mathbb{R}}(K)}(2 \pi)^{\Phi_{\mathbb{C}}(K)} h_{K} R_{K}}{w_{K} \sqrt{\left|D_{K}\right|}} \quad \text { (NF case) } ; \\
\varkappa_{K}=\frac{h_{K} r^{g}}{(r-1) \log r} \quad \text { (FF case) }
\end{gathered}
$$

(here $w_{K}$ is the number of roots of unity in $K$ ) one can see that the question about the behaviour of the ratio from the Brauer-Siegel theorem is reduced to the corresponding question for $\varkappa_{K}$. To put it into a more general framework, we first seek an interpretation of the arithmetic quantities we would like to study in terms of special values of certain zeta functions, then we study the behaviour of these special values in families using analytic methods. We will see in section 5 another applications of this principle. One also notices that this reduction step explains the appearance of the GRH in the statement of the Brauer-Siegel theorem.

Let us formulate yet another version of the generalized Brauer-Siegel theorem proven by Lebacque in [Leb07, Theorem 7]. It has the advantage of being explicit with respect to the error terms, thus giving information about the Brauer-Siegel ratio on the "finite level".

Theorem 3.5 (Lebacque). — Let $K$ be a global field. Then

(i) in the function field case

$$
\log \left(\varkappa_{K} \log r\right)=\sum_{f=1}^{N} \Phi_{r^{f}} \log \frac{r^{f}}{r^{f}-1}-\log N-\gamma+O\left(\frac{g_{K}}{N r^{N / 2}}\right)+O\left(\frac{1}{N}\right)
$$

(ii) in the number field case assuming GRH

$$
\log \varkappa_{K}=\sum_{q \leq x} \Phi_{q} \log \frac{q}{q-1}-\log \log x-\gamma+O\left(\frac{n_{K} \log x}{\sqrt{x}}\right)+O\left(\frac{g_{K}}{\sqrt{x}}\right),
$$

where $\gamma=0.577 \ldots$ is the Euler constant. The constants in $O$ are absolute and effectively computable (and, in fact, not very big).

This theorem can also be regarded as a generalization of the Mertens theorem (see [Leb07]). A slight improvement of the error term (as before, assuming GRH) was obtained in [LZ]. An unconditional number field version of this result is also available but is a little more difficult to state ([Leb07, Theorem 6]). We should also note that Lebacque's approach leads to a unified proof of the asymptotically bad and asymptotically good cases of theorem 3.2 with or without the assumption of GRH.

3.2. Limit zeta-functions. - For the moment the asymptotic theory of global fields looks like a collection of similar but not directly related results. The situation is clarified immensely by means of the introduction of limit zeta functions. 
Definition 3.6. - The limit zeta function of an asymptotically exact family of global fields $\mathcal{K}=\left\{K_{i}\right\}$ is defined as

$$
\zeta_{\mathcal{K}}(s)=\prod_{q}\left(1-q^{-s}\right)^{-\phi_{q}(\mathcal{K})},
$$

the product being taken over all prime powers in the number field case and over prime powers of the form $q=r^{f}$ in the case of curves over $\mathbb{F}_{r}$.

The basic inequalities from theorem 2.6 give the convergence of the above infinite product for $\operatorname{Re} s \geq \frac{1}{2}$ with the assumption of GRH and for Re $s \geq 1$ without it (in particular, in the function field case the infinite product converges for $\operatorname{Re} s \geq \frac{1}{2}$ ). In fact, the basic inequalities themselves can be restated in terms of the values of limit zeta functions. To formulate them we introduce the completed limit zeta function:

$$
\begin{gathered}
\tilde{\zeta}_{\mathcal{K}}(s)=e^{s} 2^{-\phi_{\mathbb{R}}} \pi^{-s \phi_{\mathbb{R}} / 2}(2 \pi)^{-s \phi_{\mathbb{C}}} \Gamma\left(\frac{s}{2}\right)^{\phi_{\mathbb{R}}} \Gamma(s)^{\phi_{\mathbb{C}}} \zeta_{\mathcal{K}}(s) \quad \text { (NF case); } \\
\tilde{\zeta}_{\mathcal{K}}(s)=r^{s} \zeta_{\mathcal{K}}(s) \quad \text { (FF case). }
\end{gathered}
$$

Let $\tilde{\xi}_{\mathcal{K}}(s)=\tilde{\zeta}_{\mathcal{K}}^{\prime}(s) / \tilde{\zeta}_{\mathcal{K}}(s)$ be the logarithmic derivative of the completed limit zeta function. Then the basic inequalities from section 2 take the following form:

Theorem 3.7 (Basic inequalities). - For an asymptotically exact family of global fields $\mathcal{K}=\left\{K_{i}\right\}$ we have $\tilde{\xi}_{\mathcal{K}}\left(\frac{1}{2}\right) \geq 0$ in the function field case and assuming GRH in the number field case and $\tilde{\xi}_{\mathcal{K}}(1) \geq 0$ without the assumption of $G R H$.

Let us give an interesting interpretation of the deficiency in terms of the distribution of zeroes of zeta functions on the critical line. In fact, the results we are going to state are interesting on their own. To a global field $K$ we associate the counting measure $\Delta_{K}=\frac{1}{g_{K}} \sum_{\rho} \delta_{t(\rho)}$, where $t(\rho)=\operatorname{Im} \rho$ in the number field case and $t(\rho)=\frac{1}{\log r} \operatorname{Im} \rho$ in the function case; the sum is taken over all zeroes $\rho$ of $\zeta_{K}(s)$ in the number field case and over all zeroes $\rho$ of $\zeta_{K}(s)$ with $t(\rho) \in(-\pi, \pi]$ in the function field case (in the case of function fields $\zeta_{K}(s)$ is periodic with the period equal to $2 \pi / \log r$ ), $\delta_{t}$ is the Dirac (atomic) measure at $t$. Thus we get a measure on $\mathbb{R}$ in the number field case and on $\mathbb{R} / \mathbb{Z}$ in the function field case. The asymptotic behaviour of $\Delta_{K}$ was first considered by Lang [Lan71] in the asymptotically bad case. The following result is proven in [TV02, Theorem 5.2] and [TV97, Theorem 2.1].

Theorem 3.8 (Tsfasman-Vlădut). - For an asymptotically exact family of global fields $\mathcal{K}=\left\{K_{i}\right\}$, assuming $G R H$, the limit $\lim _{i \rightarrow \infty} \Delta_{K_{i}}$ exists in an appropriate space of measures (to be precise, in the space of measures of slow growth on $\mathbb{R}$ in the NF case, and in the space of measures on $\mathbb{R} / \mathbb{Z}$ in the FF case). Moreover, the limit is a measure with continuous density $M_{\mathcal{K}}(t)=\operatorname{Re} \tilde{\xi}_{\mathcal{K}}\left(\frac{1}{2}+i t\right)$.

Of course, the expression for $M_{\mathcal{K}}(t)$ can be written explicitly using the invariants $\phi_{q}$. Let us note two important corollaries of the theorem. First, we get an interpretation for the 
deficiency $\delta_{K}=\tilde{\xi}_{\mathcal{K}}\left(\frac{1}{2}\right)=M_{\mathcal{K}}(0)$ as the asymptotic number of zeroes of $\zeta_{K_{i}}(s)$ accumulating at $s=\frac{1}{2}$. Second, the theorem shows that for any family of number fields zeroes of their zeta-functions get arbitrarily close to $s=\frac{1}{2}$ (and, in a sense, we even know the rate at which zeroes of $\zeta_{K_{i}}(s)$ approach to this point).

3.3. Limit zeta-functions and Brauer-Siegel type results. - Let us turn our attention to the Brauer-Siegel type results. The formulae from theorems 3.2 and 3.4 can be rewritten as $\lim _{i \rightarrow \infty} \frac{\log \varkappa_{K_{i}}}{g_{K_{i}}}=\log \zeta_{\mathcal{K}}(1)$. Furthermore, using the absolute and uniform convergence of infinite products for zeta functions for $\operatorname{Re} s>1$, Tsfasman and Vlădut prove in [TV02, Proposition 4.2] that for $\operatorname{Re} s>1$ the equality $\lim _{i \rightarrow \infty} \frac{\log \zeta_{K_{i}}(s)}{g_{K_{i}}}=\log \zeta_{\mathcal{K}}(s)$ holds. In fact, this equality remains valid for $\operatorname{Re} s<1$ (at least if we assume GRH in the number field case). The proof of the next theorem can be found in [Zy 10] in the number field case and in [Zyk11] in the function field case (where the same problem is treated in a broader context).

Theorem 3.9 (Zykin). — For an asymptotically exact family of global fields $\mathcal{K}=\left\{K_{i}\right\}$ for Re $s>\frac{1}{2}$ we have

$$
\begin{gathered}
\left.\lim _{i \rightarrow \infty} \frac{\log \left((s-1) \zeta_{K_{i}}(s)\right)}{g_{K_{i}}}=\log \zeta_{\mathcal{K}}(s) \quad \text { (NF case assuming GRH }\right) \\
\lim _{i \rightarrow \infty} \frac{\log \left(\left(r^{s}-1\right) \zeta_{K_{i}}(s)\right)}{g_{K_{i}}}=\log \zeta_{\mathcal{K}}(s) \quad(F F \text { case })
\end{gathered}
$$

The convergence is uniform on compact subsets of the half-plane $\left\{s \mid \operatorname{Re} s>\frac{1}{2}\right\}$.

The case $s=1$ of theorem 3.9 is equivalent to the Brauer-Siegel theorem and current techniques does not allow to treat it in full generality without the assumption of GRH. Thus getting unconditional results similar to theorem 3.9 looks inaccessible at the moment. The analogue of the above result for $s=\frac{1}{2}$ is considerably weaker and one has only an upper bound:

Theorem 3.10 (Zykin). - Let $\rho_{K_{i}}$ be the first non-zero coefficient in the Taylor series expansion of $\zeta_{K_{i}}(s)$ at $s=\frac{1}{2}$, i. e. $\zeta_{K_{i}}(s)=\rho_{K_{i}}\left(s-\frac{1}{2}\right)^{r_{K_{i}}+o}\left(\left(s-\frac{1}{2}\right)^{r_{K_{i}}}\right)$. Then in the function field case or in the number field case assuming that GRH is true, for any asymptotically exact family of global fields $\mathcal{K}=\left\{K_{i}\right\}$ the following inequality holds:

$$
\limsup _{i \rightarrow \infty} \frac{\log \left|\rho_{K_{i}}\right|}{g_{K_{i}}} \leq \log \zeta_{\mathcal{K}}\left(\frac{1}{2}\right) .
$$

The interest in the study of the asymptotic behaviour of zeta functions at $s=\frac{1}{2}$ is partly motivated by the corresponding problem for $L$-functions of elliptic curves over global fields, where this value is related to deep arithmetic invariants of the elliptic curves via the BirchSwinnerton-Dyer conjecture. We refer the reader to section 5 for more details. The question whether the equality holds in theorem 3.10 is rather delicate. It is related to the so called low-lying zeroes of zeta functions, that is the zeroes of $\zeta_{K}(s)$ having small imaginary part 
compared to $g_{K}$. It might well happen that the equality $\lim _{i \rightarrow \infty} \frac{\log \left|\rho_{K_{i}}\right|}{g_{K_{i}}}=\log \zeta_{\mathcal{K}}\left(\frac{1}{2}\right)$ does not hold for all asymptotically exact families $\mathcal{K}=\left\{K_{i}\right\}$ since the behaviour of low-lying zeroes is known to be rather random. Nevertheless, it might hold for "most" families (whatever it might mean).

To illustrate how hard the problem may be, let us remark that Iwaniec and Sarnak studied a similar question for the central values of $L$-functions of Dirichlet characters [IS99] and modular forms [IS00]. They manage to prove that there exists a positive proportion of Dirichlet characters (modular forms) for which the logarithm of the central value of the corresponding $L$-functions divided by the logarithm of the analytic conductor tends to zero. The techniques of the evaluation of mollified moments used in these papers are rather involved. We also note that, to our knowledge, there has been no investigation of low-lying zeroes of $L$-functions of growing degree. It seems that the analogous problem in the function field case has neither been very well studied.

Let us indicate that the corresponding question for the logarithmic derivatives of zeta functions has a negative answer. Indeed, the functional equation implies that $\lim _{i \rightarrow \infty} \frac{\zeta_{K_{i}}^{\prime}(1 / 2)}{\zeta_{K_{i}}(1 / 2)}=1$ for any family of function fields $K_{i}$. However, the logarithmic derivative of the limit zeta function $\zeta_{\mathcal{K}}(s)$ at $s=\frac{1}{2}$ equals one only for asymptotically optimal families (c.f. theorem 3.7).

As a corollary of theorem 3.9 one can obtain a result on the asymptotic behaviour of EulerKronecker constants.

Definition 3.11. - The Euler-Kronecker constant of a global field $K$ is defined as $\gamma_{K}=$ $\frac{c_{0}(K)}{c_{-1}(K)}$, where $\zeta_{K}(s)=c_{-1}(K)(s-1)^{-1}+c_{0}(K)+O(s-1)$.

In [Iha06] Y. Ihara made an extensive study of the Euler-Kronecker constants of global fields, in particular, he obtained an asymptotic formula for their behaviour in families of curves over finite fields. A complementary result in the number field setting was obtain in [Zy 10] as a corollary of theorem 3.9. In fact the theorem 3.9 gives that in asymptotically exact families the coefficients of the Laurant series at $s=1$ of the logarithmic derivatives $\zeta_{K_{i}}^{\prime}(s) / \zeta_{K_{i}}(s)$ tend to the corresponding coefficients of the Laurant series expansion of the logarithmic derivative of the limit zeta function. For zeroes coefficient this becomes:

Corollary 3.12 (Ihara-Zykin). - Assuming GRH in the number field case and unconditionally in the function field case, for any asymptotically exact family of global fields $\left\{K_{i}\right\}$ we have

$$
\lim _{i \rightarrow \infty} \frac{\gamma_{K_{i}}}{g_{K_{i}}}=-\sum_{q} \phi_{q} \frac{\log q}{q-1} .
$$

For the sake of completeness let us mention an explicit analogue of theorem 3.9 obtained in $[\mathbf{L Z}]$ :

Theorem 3.13 (Lebacque-Zykin). - For any global field $K$, any integer $N \geq 10$ and any $\epsilon=\epsilon_{0}+i \epsilon_{1}$ such that $\epsilon_{0}=\operatorname{Re} \epsilon>0$ we have 
(i) in the function field case:

$$
\sum_{f=1}^{N} \frac{f \Phi_{r}}{r^{\left(\frac{1}{2}+\epsilon\right) f}-1}+\frac{1}{\log r} \cdot Z_{K}\left(\frac{1}{2}+\epsilon\right)+\frac{1}{r^{-\frac{1}{2}+\epsilon}-1}=O\left(\frac{g_{K}}{r^{\epsilon_{0} N}}\left(1+\frac{1}{\epsilon_{0}}\right)\right)+O\left(r^{\frac{N}{2}}\right) ;
$$

(ii) and in the number field case assuming GRH:

$$
\begin{aligned}
\sum_{q \leq N} \frac{\Phi_{q} \log q}{q^{\frac{1}{2}+\epsilon}-1}+Z_{K}\left(\frac{1}{2}+\epsilon\right)+\frac{1}{\epsilon-\frac{1}{2}} & = \\
& =O\left(\frac{|\epsilon|^{4}+|\epsilon|}{\epsilon_{0}^{2}}\left(g_{K}+n_{K} \log N\right) \frac{\log ^{2} N}{N^{\epsilon_{0}}}\right)+O(\sqrt{N}) .
\end{aligned}
$$

3.4. Some other topics related to limit zeta-functions. - Let us finally state some related results on the asymptotic properties of the coefficients of zeta functions. For the moment they are only available in the function field case (see [TV97]). Let $K / \mathbb{F}_{r}(t)$ be a function field and let $\zeta_{K}(s)=\sum_{m=1}^{\infty} D_{m} r^{-m s}$ be the Dirichlet series expansion of the zeta function of $K$. One knows that $D_{m}$ is equal to the number of effective divisors of degree $m$ on the corresponding curve. We have the following results on the asymptotic behaviour of $D_{m}$ :

Theorem 3.14 (Tsfasman-Vlădut). - For an asymptotically exact family of function fields $\mathcal{K}=\left\{K_{i}\right\}$ and any real $\mu>0$ we have

$$
\lim _{i \rightarrow \infty} \frac{\log D_{[\mu g]}\left(K_{i}\right)}{g_{K_{i}}}=\min _{s \geq 1}\left(\mu s \log q+\log \zeta_{\mathcal{K}}(s)\right) .
$$

Moreover, the minimum can be evaluated explicitly via $\phi_{q}$ (c.f. [TV97, Proposition 4.1]).

Theorem 3.15 (Tsfasman-Vlădut). - For an asymptotically exact family of function fields $\mathcal{K}=\left\{K_{i}\right\}$, any $\epsilon>0$ and any $m$ such that $\frac{D_{m}}{g} \geq \mu_{1}+\epsilon$ we have

$$
\frac{\log D_{m}\left(K_{i}\right)}{h_{K_{i}}}=\frac{q^{m-g+1}}{q-1}(1+o(1))
$$

for $g \rightarrow \infty, o(1)$ being uniform in $m$. Here $\mu_{1}$ is the largest of the two roots of the equation

$$
\frac{\mu}{2}+\mu \log _{r} \frac{\mu}{2}+(2-\mu) \log _{r}\left(1-\frac{\mu}{2}\right)=-2 \log _{r} \zeta_{\mathcal{K}}(1) .
$$

We should note that $o(1)$ from theorem 3.15 is additive whereas most of the previous results were estimates of multiplicative type (they contained logarithms of the quantities in question). It would be interesting to know whether there exist analogues of the above results in the number field case.

Let us conclude by refering the reader to the Section 6 of [TV02] for a list of open questions. 


\section{Examples}

4.1. Towers of modular curves. - Let us begin with the examples of asymptotically optimal families of curves over finite fields coming from towers of modular curves. The first constructions were carried out by Ihara ([Iha81]), Tsfasman-Vlăduţ-Zink ([TVZ]). The research in this direction was continued by N. Elkies and many others. Let us describe several constructions.

4.1.1. Classical modular curves. - Let us start with the construction of towers of modular curves which leads to asymptotically optimal infinite function fields. For further information, we refer the reader to [TV92, Chapter 4]. It is well known that the modular group $\Gamma(1)=$ $\operatorname{PSL}_{2}(\mathbb{Z})$ acts on the Poincaré upper half-plane $\mathfrak{h}$ by $\left(\begin{array}{ll}a & b \\ c & d\end{array}\right) \cdot z=\frac{a z+b}{c z+d}$. We fix a positive integer $N$ and we define the principal congruence subgroup of level $N$ by

$$
\Gamma(N)=\left\{\gamma \in \Gamma(1) \mid \gamma \equiv\left(\begin{array}{ll}
1 & 0 \\
0 & 1
\end{array}\right) \quad \bmod N\right\}
$$

$\Gamma(N) \triangleleft \Gamma(1)$ and $\Gamma(1) / \Gamma(N)$ is isomorphic to $P S L_{2}(\mathbb{Z} / N \mathbb{Z})$. In particular,

$$
[\Gamma(1): \Gamma(N)]= \begin{cases}\frac{N^{3}}{2} \prod_{\ell \mid N}\left(1-\ell^{-2}\right) & \text { si } N \geq 3 \\ 6 & \text { si } N=2 .\end{cases}
$$

We also put $\Gamma_{0}(N)=\left\{\gamma \in \Gamma(1) \mid \gamma \equiv\left(\begin{array}{ll}* & * \\ 0 & *\end{array}\right) \bmod N\right\}$, so that $\Gamma(N) \subset \Gamma_{0}(N)$. We have $\left[\Gamma(1): \Gamma_{0}(N)\right]=N \prod_{\ell \mid N}\left(1-\ell^{-1}\right)$.

Let now $\Gamma$ be a congruence subgroup, that is, any subgroup of $\Gamma(1)$ containing $\Gamma(N)$. The most important case for us is $\Gamma=\Gamma(N)$ or $\Gamma_{0}(N)$. The set $Y_{\Gamma}=\Gamma \backslash \mathfrak{h}$ is equipped with an analytic structure, but is not compact. To compactify it we add points at infinity (named cusps): $\Gamma(1)$ acts naturally on $\mathbb{P}^{1}(\mathbb{Q})$ and we put $X_{\Gamma}=(\Gamma \backslash \mathfrak{h}) \cup\left(\Gamma \backslash \mathbb{P}^{1}(\mathbb{Q})\right)$. This way it becomes a connected Riemann surface called modular curve. We let $X(N)=X_{\Gamma(N)}, X_{0}(N)=X_{\Gamma_{0}(N)}$, $Y(N)=Y_{\Gamma(N)}$ and $Y_{0}(N)=X_{\Gamma_{0}(N)}$.

If $\Gamma^{\prime} \subset \Gamma \subset \Gamma(1)$, there is a natural projection from $X_{\Gamma^{\prime}} \rightarrow X_{\Gamma}$, which allows us to compute the genus of the modular curve using the covering (the function $j$ is in fact the $j$-invariant of the elliptic curve $\mathbb{C} /(\mathbb{Z}+z \mathbb{Z}))$ :

$$
X_{\Gamma} \longrightarrow X_{\Gamma(1)} \underset{j}{\sim} \mathbb{P}^{1}(\mathbb{C})
$$

via the Hurwitz formula. For instance,

$$
g_{X(N)}=1+\frac{(N-6)[\Gamma(1): \Gamma(N)]}{12 N} .
$$


It can be shown that $Y(1)$ classifies isomorphism classes of complex elliptic curves and that $Y_{0}(N)$ classifies pairs $\left(E, C_{N}\right), E$ being a complex elliptic curve and $C_{N}$ being a cyclic subgroup of $E$ of order $N$.

Now, to construct towers of curves defined over finite fields, we need to take reductions of our modular curves modulo primes. If $S$ is a scheme and $E \rightarrow S$ is an elliptic curve, the set of sections $E(S)$ is an abelian group. Let $E_{N}(S)$ denote the points of order dividing $N$ in $E(S)$. We call a level $N$ structure an isomorphism $\alpha_{N}: E_{N}(S) \rightarrow(\mathbb{Z} / N \mathbb{Z})^{2}$. One can prove that there exists a smooth affine scheme $Y(N)$ over Spec $\mathbb{Z}[1 / N]$ classifying the isomorphism classes of pairs $\left(E, \alpha_{N}\right)$ consisting of an elliptic curve $E / \operatorname{Spec} \mathbb{Z}[1 / N]$ together with a level $N$ structure $\alpha_{N}$ on $E$. One can prove that this curve is a model of $Y(N)$ over $\operatorname{Spec} \mathbb{Z}\left[\zeta_{N}, 1 / N\right]$, where $\zeta_{N}$ is a primitive $N^{\text {th }}$-root of 1 . There is also a model of $Y_{0}(N)$ over Spec $\mathbb{Z}[1 / N]$ and this "coarse" moduli space classifies pairs consisting of an elliptic curve together with a cyclic subgroup of order $N$. Models for $X(N)$ and $X_{0}(N)$ can also be obtained in such a way that they become compatible with those for $Y(N)$ and $Y_{0}(N)$. These curves have good reduction over any prime ideal not dividing $N$. Moreover, the curve $X_{0}(N)$ can be defined over $\mathbb{Q}$ and has good reduction at any prime number not dividing $N$. Let $p$ be such prime. We denote by $C_{0, N}$ the curve over $\mathbb{F}_{p^{2}}$ obtained by reduction of $X_{0}(N) \bmod p$. The curve $X(N)$ can be defined over the quadratic subfield of $\mathbb{Q}\left(\zeta_{N}\right)$ and has good reduction at all the primes not dividing $N$. Let $C_{N}$ be the reduction of $X(N)$ at a prime, i. e. a curve over $\mathbb{F}_{p^{2}}$. One can see that the genus of $X_{0}(N)$ and of $X(N)$ is preserved under reduction. The points

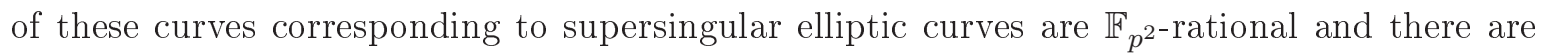
$\frac{[\Gamma(1): \Gamma(N)]}{12}(p-1)$ of them on $C_{N}$. This leads to the following theorem:

Theorem 4.1. - (Ihara, Tsfasman-Vlădut-Zink) Let $\ell$ be a prime number not equal to $p$. The families $\left\{C_{\ell^{n}}\right\}$ and $\left\{C_{0, \ell^{n}}\right\}$ satisfy $\phi_{p^{2}}=p-1$ and therefore are asymptotically optimal.

Note that the result for $C_{0, \ell^{n}}$ can be deduced immediately from the corresponding result for $C_{\ell^{n}}$.

4.1.2. Shimura modular curves. - Similar results on Shimura curves allow us to construct directly asymptotically optimal families over $\mathbb{F}_{r}$ with $r=q^{2}=p^{2 m}, p$ prime. To do so, following Ihara, we start with a $\mathfrak{p}$-adic field $k_{\mathfrak{p}}$ with $N(\mathfrak{p})=q=p^{m}$. Let $\Gamma$ be a torsion-free discrete subgroup of $G=P S L_{2}(\mathbb{R}) \times P S L_{2}\left(k_{\mathfrak{p}}\right)$ with compact quotient and dense projection to each of the two components of $G$ (such $\Gamma$ 's exist). Ihara proved the following results that relate the construction of optimal curves to (anabelian) class field theory, and therefore are of great interest for us:

Theorem 4.2. - (Ihara [Iha08]) To any subgroup $\Gamma$ of $G$ with the above properties one can associate a complete smooth geometrically irreducible curve $X$ over $\mathbb{F}_{r}$ of genus $\geq 2$, together with a set $\Sigma$ consisting of $(q-1)(g-1) \mathbb{F}_{r}$-rational points of $X$ such that there is a canonical isomorphism (up to conjugacy) from the profinite completion of $\Gamma$ to $\operatorname{Gal}\left(K^{\Sigma} / K\right)$ where $K^{\Sigma}$ 
denotes the maximal unramified Galois extension of the function field $K$ of $X$ in which all the places corresponding to the points of $\Sigma$ are completely split.

An easy computation leads to the following result:

Corollary 4.3. - For any square prime power $r$, there is a tower of curves defined over $\mathbb{F}_{r}$ with $\phi_{r}=\sqrt{r}-1$.

In fact, the elliptic modular curves $X(N)$ that we constructed in the previous section correspond to $\Gamma=P S L_{2}(\mathbb{Z}[1 / p])$ and its principal congruence subgroups of level $N$.

4.1.3. Drinfeld modular curves. - The applicability of Drinfeld modular curves to the problem of construction of optimal curves has been known since late 80's. The results we are going to discuss next can be found in [TV92].

Let $L$ be a field of characteristic $p$ and let $L\{\tau\}$ denote the ring of non-commutative polynomials in $\tau$, consisting of expressions of the form $\sum_{i=0}^{n} a_{i} \tau^{i}, a_{i} \in L$, with multiplication satisfying $\tau \cdot a=a^{p} \cdot \tau$ for any $a \in L$. Let $A=\mathbb{F}_{r}[T]$.

A Drinfeld module is an $\mathbb{F}_{r}$-homomorphism $\phi: A \rightarrow L\{\tau\}, a \mapsto \phi_{a}$ satisfying a few technical conditions. Let $\gamma$ be the map $\gamma: A \rightarrow L$ sending $a \in A$ to the term of $\phi_{a}$ of degree zero. Notice that $\phi$ is determined by $\phi_{T}$ and $\gamma$ by $\gamma(T)$. We consider only Drinfeld modules of rank 2 that is we assume that $\phi_{T}$ is a polynomial in $\tau$ of degree 2 and we put $\phi_{T}=\gamma(T)+g \tau+\Delta \tau^{2}$ $(\Delta \neq 0)$. More generally, one can define Drinfeld modules over any $A$-scheme $S$.

Just as in the classical case, given a proper ideal $I$ of $A$, one can define a level $I$ structure on $\phi$. There is an affine scheme $M(I)$ of finite type over $A$ that parametrizes pairs $(\phi, \lambda)$, where $\phi$ is a Drinfeld module over $S$ and $\lambda$ is a level $I$ structure. The scheme $M(I)$ has a canonical compactification: there exists a unique scheme $\overline{M(I)}$ containing $M(I)$ as an open dense subscheme, whose fibres over Spec $A\left[I^{-1}\right]$ are smooth complete curves. The group $\mathrm{GL}_{2}(A / I)$ acts naturally on $M(I)$ by operating on the structures of level $I$ and this action extends to $\overline{M(I)}$.

From now on, let $I$ be a prime ideal generated by a polynomial of degree $m$ prime to $q-1$. Now, consider the smooth complete (reducible) curve $X(I)=\overline{M(I)} \otimes_{A} \mathbb{F}_{q}$ over $\mathbb{F}_{q}$. Note that the $A$-algebra structure on $\mathbb{F}_{q}$ is obtained through the reduction mod $T$. Consider the subgroup

$$
\Gamma_{0}(I)=\left\{\left(\begin{array}{ll}
a & b \\
c & d
\end{array}\right) \in G L_{2}(A) \mid c \in I\right\}
$$

and let $\overline{\Gamma_{0}(I)}$ be the image of this subgroup in $\mathrm{GL}_{2}(A / I)$. Finally, we consider the smooth complete absolutely irreducible curve $X_{0}(I)=X(I) / \overline{\Gamma_{0}(I)}$. The image of $\overline{M(I)}-M(I)$ in $X_{0}(I)$ consists of two $\mathbb{F}_{q}$-rational points. Moreover, the following result holds.

Theorem 4.4. - The family $\left\{X_{0}(I)\right\}$, where $I$ is a prime ideal of A generated by a polynomial of degree prime to $q-1$, is an asymptotically exact family of curves defined over $\mathbb{F}_{q}$, satisfying $\phi_{q^{2}}=q-1$ and thus is optimal. 
Moreover, N. Elkies proved in [Elk] that the family of curves $\dot{X}_{0}\left(T^{n}\right)$ which parametrizes normalized Drinfeld modules $(\gamma(T)=1, \Delta=-1)$ with a level $T^{n}$ structure is asymptotically optimal. He also related it to the explicit towers of Garcia and Stichtenoth discussed in the next subsection.

4.2. Explicit towers. - In the last fifteen years, Garcia, Stichtenoth and many others managed to construct asymptotically good towers explicitely in a recursive way. Their interest comes from coding theory for such towers provide asymptotically good codes via the construction of Goppa. Let us give an example of such explicit towers.

Theorem 4.5. - (Garcia-Stichtenoth) Let $r=q^{2}$ be a prime power. The tower $\left\{F_{n}\right\}$ defined recursively starting from the rational function field $F_{0}=\mathbb{F}_{r}\left(x_{0}\right)$ using the relations $F_{n+1}=$ $F_{n}\left(x_{n+1}\right)$, where

$$
x_{n+1}^{q}+x_{n+1}=\frac{x_{n}^{q}}{x_{n}^{q-1}+1},
$$

satisfies $\phi_{r}=\sqrt{r}-1$ and thus is optimal.

If the cardinality of the ground field is not a square no towers with $\phi_{r}=\sqrt{r}-1$ are known. However, there exist optimal towers in the sense that they have zero deficiency. Such towers can be constructed starting from an explicit tower over a bigger field using a descent argument (see Ballet-Rolland [BR] for the details) or using modular towers.

Let us now say a word about Elkies modularity conjecture. Elkies' work shows that most of the recursive examples of Garcia and Stichtenoth can be obtained by finding equations for suitable modular towers. This made him formulate the following conjecture:

Conjecture 4.6 (Elkies). - Any asymptotically optimal tower is modular.

Finally, let us note that there are other interesting constructions leading to explicit asymptotically good towers of function fields. As an example we mention the paper [BB] by P. Beelen and I. Bouw who use Fuchsian differential equations to produce optimal towers over $\mathbb{F}_{q^{2}}$.

4.3. Classfield towers. - As it was said in section 2, tamely ramified infinite extensions of global fields with finitely many ramified places and with completely split places give examples of asymptotically good towers. Given a global field $K$, it is natural to consider the maximal extension of $K$ unramified outside a finite set of places $S$, in which places from a set $T$ are completely split. But these extensions are very hard to understand. The maximal $\ell$-extensions are much easier to handle. These extensions are the limits of the $\ell-S$ - $T$-class field towers of K.

For a global field $K$, two sets of finite places $S$ and $T(T \neq \emptyset(F F))$ of $K$, and a prime number $\ell$, consider the maximal abelian $\ell$-extension $H_{S, \ell}^{T}(K)$ of $K$, unramified outside $S$ and in which the places from $T$ are split (in the case of function fields the assumption on $T$ to be non-empty is made in order to avoid infinite constant field extensions). Consider the tower recursively constructed as follows: $K_{0}=K, K_{i+1}=H_{S, \ell}^{T}\left(K_{i}\right)$. All the extensions $K_{i} / K$ are Galois, 
and we denote by $G_{S}^{T}(K, \ell)$ the Galois group $\operatorname{Gal}\left(\bigcup_{i} K_{i}, K\right)$. A sufficient condition for this tower to be infinite is given by the Golod-Shafarevich theorem: if $G$ is a finite $\ell$-group then $\operatorname{dim}_{\mathbb{F}_{\ell}} H^{2}\left(G, \mathbb{F}_{\ell}\right)>\frac{1}{4} \operatorname{dim}_{\mathbb{F}_{\ell}} H^{1}\left(G, \mathbb{F}_{\ell}\right)^{2}$. This allows to construct asymptotically good infinite global fields. The following result is at the base of many constructions of class field towers with prescribed properties:

Theorem 4.7. - [Tsfasman-Vlădut [TV02] (NF), Serre [Ser85], Niederreiter-Xing [NX] $(F F)]$ Let $K / k$ be a cyclic extension of global fields of degree $\ell$. Let $T(k)$ be a finite set of non archimedean places of $k$ and let $T(K)$ be the set of places above $T(k)$ in $K$. Suppose in the function field case that $\operatorname{GCD}\{\ell, \operatorname{deg} \mathfrak{p}, \mathfrak{p} \in T(K)\}=1$. Let $Q$ be the ramification locus of $K / k$. Let

$$
\begin{aligned}
(F F) \quad C(T, K / k)= & \# T(k)+2+\delta_{\ell}+2 \sqrt{\# T(K)+\delta_{\ell}}, \\
(N F) \quad C(T, K / k)= & \# T(K)-t_{0}+r_{1}+r_{2}+\delta_{\ell}+2-\rho+ \\
& 2 \sqrt{\# T(K)+\ell\left(r_{1}+r_{2}-\rho / 2\right)+\delta_{\ell}},
\end{aligned}
$$

where $\delta_{\ell}=1$ if $K$ contains the $\ell$-root of unity, and 0 otherwise, $t_{0}$ is the number of principal ideals in $T(k), r_{1}=\Phi_{\mathbb{R}}(K), r_{2}=\Phi_{\mathbb{C}}(K)$ and $\rho$ is the number of real places of $k$ which become complex in $K$. Suppose that $\# Q \geq C(T, K / k)$. Then $K$ admits an infinite unramfied $\ell-T(K)$-class field tower.

One can construct such cyclic extension using the Grunwald-Wang theorem (and sometimes even explicitly by hand) and deduce the following result:

Corollary 4.8 (Lebacque). - Let $n$ be an integer and let $t_{1}, \ldots, t_{n}$ be prime powers (NF) (powers of $p(F F))$. There exists an infinite global field (both in the number field and function field cases) such that $\phi_{t_{1}}, \ldots, \phi_{t_{n}}$ are all $>0$.

Another way to produce asymptotically good infinite class field towers is to use tamely ramified instead of unramified class field towers. This is the subject of [HM01] and [HM02].

The question of finding asymptotically good towers with given Tsfasman-Vlădut invariants equal to zero is more difficult. A related question is to find out whether an infinite global extension realizes the maximal local extension at a given prime. Using results of J. Labute [Lab] and A. Schmidt [Sch], the following theorem is proven:

Theorem 4.9 (Lebacque [Leb09]). - Let $P=\left\{\mathfrak{p}_{1}, \ldots, \mathfrak{p}_{n}\right\} \subset P l_{f}(\mathcal{Q})$. Assume that for any $i=1, \ldots, n$ we have $n_{i}$ distinct positive integers $d_{i, 1}, \ldots, d_{i, n_{i}}$. Let $I \subset P l_{f}(\mathcal{Q})$ be a finite set of finite places of $\mathcal{Q}$ such that $I \cap P=\emptyset$. There exists an infinite global field $\mathcal{K}$ such that:

1. $I \cap \operatorname{Supp}(\mathcal{K})=\emptyset$,

2. For any $i=1, \ldots, n$, and any $j=1, \ldots, n_{i}, \quad \phi_{\mathfrak{p}_{i}, \mathrm{~Np}_{i}}{ }_{i, j}=\frac{\phi_{\infty}}{n_{i} d_{i, j}}>0$.

3. One can explicitly estimate $\phi_{\infty}$ and the deficiency in terms of $P, I, n_{i}$ and $d_{i j}$.

The $\phi_{\mathfrak{p}, q}$ are invariants generalizing the classical $\phi_{q}$ : they count the asymptotic number of primes of norm $q$ above a given prime $\mathfrak{p}$ (see [Leb10] for a definition). In the case of $\mathbb{Q}$ they 
coincide with the classical ones. This extension is obtained as the compositum of a finite extension of $\mathcal{Q}$ with prescribed positive $\Phi_{\mathfrak{p}_{i}, \mathrm{~Np}_{i}}{ }_{i, j}>0$ and an infinite class field tower $\mathcal{Q}_{S}^{P}(\ell)$ satisfying the $K(\pi, 1)$ property of A. Schmidt.

4.4. Bounds on the deficiency. - We have already seen that, using towers of modular curves, one can produce infinite function fields over $\mathbb{F}_{r}$ with zero deficiency. If $r$ is a square, there are even towers with $\phi_{r}=\sqrt{r}-1$. In the case of number fields no zero deficiency infinite number fields are known. In fact we doubt that the class field theory (which is for now the only method to produce asymptotically good infinite number fields) can ever give such field. Let us quote here the example with the smallest known deficiency due to F. Hajir and Ch. Maire [HM02].

Let $k=\mathbb{Q}(\xi)$, where $\xi$ is a root of $f(x)=x^{6}+x^{4}-4 x^{3}-7 x^{2}-x+1$. Consider the element $\eta=-671 \xi^{5}+467 \xi^{4}-994 \xi^{3}+3360 \xi^{2}+2314 \xi-961 \in \mathcal{O}_{k}$. Let $K=k(\sqrt{\eta})$. F. Hajir and Ch. Maire prove using a Golod-Shafarevich like result that $K$ admits an infinite tamely ramified tower satisfying $\delta \leq 0.137 \ldots$

\section{Higher dimensional theory}

In this section we will mostly consider the function field case since most of the results we are going to mention are unavailable in the number field case. However, we will give some references to the number field case as well.

5.1. Number of points on higher dimensional varieties. - The question about the maximal number of points on curves over finite fields has been extensively studied by numerous authors. The analogous question for higher dimensional varieties has received comparatively little attention most probably due to its being significantly more difficult.

As for the curves, we have the so-called Weil bound which is in this case a famous theorem of Deligne. Similarly, this bound is not optimal and the general framework for improving it is provided by the explicit formulae. In the case of curves over $\mathbb{F}_{r}$ Oesterle managed to find the best bounds available through the techniques of explicit formulae for any given $r \neq 2$ (see [Ser85]). A decade later the case of arbitrary varieties over finite fields was treated by $\mathrm{G}$. Lachaud and M. A. Tsfasman in [Tsf95] and [LT]. Let us reproduce here the main results from $[\mathbf{L T}]$. To do so we will have to introduce some notation concerning varieties over finite fields.

Let $X$ be a non-singular absolutely irreducible projective variety of dimension $d$ defined over a finite field $\mathbb{F}_{r}$. We put $X_{f}=X \otimes_{\mathbb{F}_{r}} \mathbb{F}_{q^{r}}$ and $\bar{X}=X \otimes_{\mathbb{F}_{r}} \overline{\mathbb{F}}_{r}$. Let $\Phi_{r f}=\Phi_{r f}(X)$ be the number of points of $X$ having degree $f$. Thus, for the number $N_{f}$ of $\mathbb{F}_{r}$-points of the variety $X_{f}$ we have the formula $N_{f}=\sum_{m \mid f} m \Phi_{r^{m}}$. We denote by $b_{s}(X)=\operatorname{dim}_{\mathbb{Q}_{l}} H^{s}\left(\bar{X}, \mathbb{Q}_{l}\right)$ the $l$-adic Betti numbers of $X$.

The family of inequalities proven in $[\mathbf{L T}]$ has a doubly positive sequence as a parameter. Let us introduce the corresponding notation. To a sequence of real numbers $\mathbf{v}=\left(v_{n}\right)_{n \geq 0}$ we 
associate the family of power series $\psi_{m, \mathbf{v}}(t)=\sum_{n=1}^{\infty} v_{m n} t^{n}$. We denote $\psi_{\mathbf{v}}(t)=\psi_{1, \mathbf{v}}(t)$ and let $\rho_{\mathbf{v}}$ be the radius of convergence of this power series. A doubly positive sequence $\mathbf{v}$ is such a sequence that $0 \leq v_{n} \leq v_{0}$ for all $n, v_{0}=1$ and for any $z \in \mathbb{C},|z|<1$ we have $1+2 \operatorname{Re} \psi_{\mathbf{v}}(t) \geq 0$.

We will also need the functions $F_{m, \mathbf{v}}(k, t)=\sum_{s=0}^{\infty}(-1)^{s} \psi_{m, \mathbf{v}}\left(r^{-k s} t\right)=\sum_{n=1}^{\infty} \frac{v_{m n} t^{m n}}{1+r^{-m n k}}, F_{\mathbf{v}}(k, t)=$ $F_{1, \mathbf{v}}(k, t)$. We let $A_{\mathbf{v}}(z)=-\min _{|t|=z} \operatorname{Re} \psi_{\mathbf{v}}(t)$ and denote $I(k)=\{i \mid 1 \leq i \leq 2 d-1, i \neq k, i \neq$ $2 d-k\}$ the set of indices. We have the following inequalities:

Theorem 5.1 (Lachaud-Tsfasman). - For any odd integer $k, 1 \leq k \leq d$, any doubly positive sequence $\mathbf{v}=\left(v_{n}\right)_{n \geq 0}$ with $\rho_{\mathbf{v}}>q^{k / 2}$ and any $M \geq 1$ we have

$$
\begin{aligned}
\sum_{m=1}^{M} m \Phi_{r^{m}}(X) \psi_{m, \mathbf{v}}\left(r^{-(2 d-k) / 2}\right) \leq \psi_{\mathbf{v}}\left(r^{-(2 d-k) / 2}\right)+\psi_{\mathbf{v}}\left(r^{k / 2}\right)+\frac{b_{k}}{2}+ & \\
& +\sum_{i \text { odd }, i \neq k} b_{i} A_{\mathbf{v}}\left(r^{-(i-k) / 2}\right)+\sum_{i \text { even }} b_{i} \psi_{\mathbf{v}}\left(r^{-(i-k) / 2}\right),
\end{aligned}
$$

and

$$
\begin{aligned}
\sum_{m=1}^{M} m \Phi_{r^{m}}(X) F_{m, \mathbf{v}}\left(d-k, r^{-(2 d-k) / 2}\right) \leq F_{\mathbf{v}}\left(d-k, r^{-(2 d-k) / 2}\right) & +F_{\mathbf{v}}\left(d-k, r^{k / 2}\right)+ \\
& +\frac{b_{k}}{2}+\sum_{i \in I(k)} b_{i} F_{\mathbf{v}}\left(d-k, r^{-(i-k) / 2}\right) .
\end{aligned}
$$

For example, taking the second inequality with $\psi_{\mathbf{v}}(t)=\frac{t}{2}$ we get the classical Weil bound, taking the first one with $\psi_{\mathbf{v}}(t)=\frac{t}{1-t}$ we get (asymptotically) a direct generalization of the Drinfeld-Vlădut bounds. These inequalities are not straightforward to apply. We refer the reader to $[\mathbf{L T}]$ for more details on how to make good choices of the doubly positive sequence. Unfortunately, in the case of dimension $d \geq 2$ the optimal choice of $\mathbf{v}$ is unknown.

The asymptotic versions of these inequalities can be easily deduced from theorem 5.1 once one introduces proper definitions. For a variety $X$ let $b(X)=\max _{i=0, \ldots, d} b_{i}(X)$ be the maximum of its $l$-adic Betti numbers.

Definition 5.2. - A family of varieties $\left\{X_{j}\right\}$ is called asymptotically exact if the limits $\phi_{r f}=\lim _{j \rightarrow \infty} \frac{\Phi_{r}\left(X_{j}\right)}{b\left(X_{j}\right)}$ and $\beta_{i}=\lim _{j \rightarrow \infty} \frac{b_{i}\left(X_{j}\right)}{b\left(X_{j}\right)}$ exist. It is asymptotically good if at least one of $\phi_{r f}$ is different from zero.

We can state the following corollary of theorem 5.1:

Corollary 5.3. - In the notation of theorem 5.1 for an asymptotically exact family of varieties one has 


$$
\sum_{m=1}^{M} m \phi_{r^{m}} \psi_{m, \mathbf{v}}\left(r^{-(2 d-k) / 2}\right) \leq \frac{\beta_{k}}{2}+\sum_{i \text { odd }, i \neq k} \beta_{i} A_{\mathbf{v}}\left(r^{-(i-k) / 2}\right)+\sum_{i \text { even }} \beta_{i} \psi_{\mathbf{v}}\left(r^{-(i-k) / 2}\right),
$$

and

$$
\sum_{m=1}^{M} m \phi_{r^{m}} F_{m, \mathbf{v}}\left(d-k, r^{-(2 d-k) / 2}\right) \leq \frac{\beta_{k}}{2}+\sum_{i \in I(k)} \beta_{i} F_{\mathbf{v}}\left(d-k, r^{-(i-k) / 2}\right) .
$$

Taking particular examples of the sequence $\mathbf{v}$ one gets more tractable inequalities (see [LT]).

5.2. Brauer-Siegel type conjectures for abelian varieties over finite fields. - One can ask about the possibility of extending the Brauer-Siegel theorem to the case of varieties over finite fields. The question is not as easy as it might seem. First, mimicking the proof of theorem 3.4 one gets a result about the asymptotic behaviour of the residues of zeta functions of varieties at $s=d$ (see [Zyk09]). Such a result would be interesting if there was a reasonable interpretation for this residue in terms of geometric invariants of our variety.

Two other approaches were suggested by B. Kunyavskii and M. Tsfasman and by M. Hindry and A. Pacheco. Both of them have for their starting points the Birch and Swinnerton-Dyer (BSD) conjecture which expresses the value at $s=1$ of the $L$-function of an abelian variety in terms of certain arithmetic invariants related to this variety. However, the situation with the asymptotic behaviour of this special value of the $L$-functions is much less clear than before. Let us begin with the approach of Kunyavskii and Tsfasman.

Let $K / \mathbb{F}_{r}$ be a function field and let $A / K$ be an abelian variety over $K$. We denote by $\amalg_{A}:=|\amalg(A / K)|$ the order of the Shafarevich-Tate group of $A$, and by $\operatorname{Reg}_{A}$ the determinant of the Mordell-Weil lattice of $A$ (see $[\mathbf{H P}]$ for definitions). Note that in a certain sense $\amalg_{A}$ and $\operatorname{Reg}_{A}$ are the analogues of the class number and of the regulator respectively. Kunyavskii and Tsfasman make the following conjecture concerning families of constant abelian varieties (see $[\mathbf{K T}])$ :

Conjecture 5.4. - Let $A_{0}$ be a fixed abelian variety over $\mathbb{F}_{r}$. Take an asymptotically exact family of function fieds $\mathcal{K}=\left\{K_{i}\right\}$ and put $A_{i}=A_{0} \times_{\mathbb{F}_{r}} K_{i}$. Then

$$
\lim _{i \rightarrow \infty} \frac{\log _{r}\left(\amalg_{i} \cdot \operatorname{Reg}_{i}\right)}{g_{i}}=1-\sum_{m=1}^{\infty} \phi_{r^{m}}(\mathcal{K}) \log _{r} \frac{\left|A_{0}\left(\mathbb{F}_{r^{m}}\right)\right|}{r^{m}} .
$$

This conjecture is actually stated as theorem in $[\mathbf{K T}]$. Unfortunately the change of limits in the proof given in [KT] is not justified thus the proof can not be considered a valid one. In fact the flaw looks very difficult to repair as the statement of the theorem can be reduced (via a formula due to J. Milne, which gives the BSD conjecture in this case) to an equality of the type $\lim _{i \rightarrow \infty} \frac{\log \zeta_{K_{i}}(s)}{g_{K_{i}}}=\log \zeta_{\mathcal{K}}(s)$ at a given point $s \in \mathbb{C}$ with $\operatorname{Re} s=\frac{1}{2}$ (in fact $s$ belongs to a finite set of points depending on $A_{0}$ ). As we have already mentioned in the discussion following theorem 3.10 this question does not look accessible at the moment. 
Let us turn our attention to the approach of Hindry and Pacheco. They treat the case in some sense "orthogonal" to that of Kunyavskii and Tsfasman. Here is the conjecture they make in [HP]:

Conjecture 5.5. - Consider the family $\left\{A_{i}\right\}$ of non-constant abelian varieties of fixed dimension over the fixed function field $K$. We have

$$
\lim _{i \rightarrow \infty} \frac{\log \left(\amalg_{i} \cdot \operatorname{Reg}_{i}\right)}{\log H\left(A_{i}\right)}=1,
$$

where $H\left(A_{i}\right)$ is the exponential height of $A_{i}$.

Using deep arguments from the theory of abelian varieties over function fields the conjecture is reduced in $[\mathbf{H P}]$ to the one on zeroes of $L$-functions of abelian varieties together with the BSD conjecture. Hindry and Pacheco are actually faced with the problem of the type discussed after theorem 3.10, this time for abelian varieties over function fields.

The following example serves as the evidence for the last conjecture (see $[\mathbf{H P}]$ ):

Theorem 5.6 (Hindry-Pacheco). - For the family of elliptic curves $E_{d}$ over $\mathbb{F}_{r}(t)$, where the characteristic of $\mathbb{F}_{r}$ is not equal to 2 or 3 , defined by the equations $y^{2}+x y=x^{3}-t^{d}, d \geq 1$ and prime to $r$, the Tate-Shafarevich group $\amalg\left(E_{d} / K\right)$ is finite and

$$
\log \left(\amalg_{d} \cdot \operatorname{Reg}_{d}\right) \sim \log H\left(E_{d}\right) \sim \frac{d \log r}{6} .
$$

The proof of this theorem uses a deep result of Ulmer [Ulm02] who established the BSD conjecture in this case and explicitly computed the $L$-functions of $E_{d}$. This reduces the statement of the theorem to a an explicit (though highly non-trivial) estimate involving Jacobi sums.

The conjectures 5.4 and 5.5 can be united (though not proved) within the general asymptotic theory of $L$-functions over function fields. Such a theory also explains why we get 1 as a limit in the second conjecture and a complicated expression in the first one. We will sketch some aspects of the theory in the next subsection.

The analogous problem in the number field case has also been considered [Hin]. Unfortunately in the number field case we do not have a single example supporting the conjecture.

5.3. Asymptotic theory of zeta and $L$-functions over finite fields. - The proofs of the results from this subsection as well as lengthy discussions can be found in [Zyk11]. Let us first define axiomatically the class of functions we are going to work with. This resembles the so called Selberg class from the analytic number theory, but, of course the case of function fields is infinitely easier from the analytic point of view, all functions being rational (or even polynomial).

Definition 5.7. - An $L$-function $L(s)$ over a finite field $\mathbb{F}_{r}$ is a holomorphic function in $s$ such that for $u=q^{-s}$ the function $\mathcal{L}(u)=L(s)$ is a polynomial with real coefficients, $\mathcal{L}(0)=1$ and all the roots of $\mathcal{L}(u)$ are on the circle of radius $r^{-\frac{d}{2}}$ for some non-negative integer number $d$ which is called the weight of the $L$-function. We say that the degree of the polynomial $\mathcal{L}(u)$ 
is the degree of the corresponding $L$-function. A zeta function $\zeta(s)$ is a product of $L$-functions in powers \pm 1 :

$$
\zeta(s)=\prod_{k=0}^{d} L_{k}(s)^{w_{k}}
$$

where $w_{k} \in\{-1,1\}$ and $L_{k}(s)$ is an $L$-function of weight $k$.

Both zeta-functions of smooth projective curves or even varieties over finite fields and $L$ functions of elliptic surfaces considered in the previous sections are covered by this definition. For the logarithm of a zeta function we have the Dirichlet series expansion:

$$
\log \zeta(s)=\sum_{f=1}^{\infty} \frac{\Lambda_{f}}{f} r^{-f s}
$$

which is convergent for $\operatorname{Re} s>\frac{d}{2}$. In the case of a variety $X / \mathbb{F}_{r}$ we have a simple interpretation for the coefficients $\Lambda_{f}=\left|X\left(\mathbb{F}_{r} f\right)\right|$ as the number of points on $X$ over the degree $f$ extension of $\mathbb{F}_{r}$.

We are going to work with zeta and $L$-functions asymptotically, so we have to introduce the notion of a family. We will call a sequence $\left\{\zeta_{k}(s)\right\}_{k=1 \ldots \infty}=\left\{\prod_{i=0}^{d} L_{k i}(s)^{w_{i}}\right\}_{k=1 \ldots \infty}$ of zeta functions a family if the total degree $g_{k}=\sum_{i=0}^{d} g_{k i}$ tends to infinity and $d$ remains constant. Here $g_{k i}$ are the degrees of the individual $L$-functions $L_{k i}(s)$ in $\zeta_{k}(s)$.

Definition 5.8. - A family $\left\{\zeta_{k}(s)\right\}_{k=1 \ldots \infty}$ of zeta-functions is called asymptotically exact if the limits

$$
\gamma_{i}=\lim _{k \rightarrow \infty} \frac{g_{k i}}{g_{k}} \text { and } \lambda_{f}=\lim _{k \rightarrow \infty} \frac{\Lambda_{k f}}{g_{k}}
$$

exist for each $i=0, \ldots, d$ and each $f \in \mathbb{Z}, f \geq 1$. The family is called asymptotically bad if $\lambda_{f}=0$ for any $f$ and asymptotically good otherwise.

In the case of curves over finite fields the denominators of zeta functions are negligible from the asymptotic point of view. In general we give the following definition:

Definition 5.9. - Let $\left\{\zeta_{k}(s)\right\}$ be an asymptotically exact family of zeta functions. Define the set $I \subset\{0 \ldots d\}$ by the condition $i \in I$ if and only if $\gamma_{i}=0$. We define $\zeta_{\mathbf{n}, k}(s)=\prod_{i \in I} L_{k i}(s)^{w_{i}}$ the negligible part of $\zeta_{k}(s)$ and $\zeta_{\mathbf{e}, k}(s)=\prod_{i \in\{0, \ldots, d\} \backslash I} L_{k i}(s)^{w_{i}}$ the essential part of $\zeta_{k}(s)$. Define also $d_{\mathbf{e}}=\max \{i \mid i \notin I\}$.

Definition 5.10. - We say that an asymptotically exact family of zeta or $L$-functions is asymptotically very exact if the series

$$
\sum_{f=1}^{\infty}\left|\lambda_{f}\right| q^{-\frac{f d_{\mathbf{e}}}{2}}
$$


is convergent.

In the case of curves or varieties the positivity of $\Lambda_{f}$ automatically implies the fact that the corresponding family is asymptotically very exact. This is of course false in general (an obvious example of a family which is asymptotically exact but not very exact is given by $\left.L_{k}(s)=\left(1-q^{-s}\right)^{k}\right)$. In general most of the results are proven for asymptotically very exact families and not just for asymptotically exact ones.

We have already noted that the concept of limit zeta functions is of utmost importance in the asymptotic theory.

Definition 5.11. - Let $\left\{\zeta_{k}(s)\right\}$ be an asymptotically exact family of zeta functions. Then the corresponding limit zeta function is defined as

$$
\zeta_{\lim }(s)=\exp \left(\sum_{f=1}^{\infty} \frac{\lambda_{f}}{f} q^{-f s}\right) .
$$

Now, we can state the generalizations of most of the results concerning zeta and $L$-functions over finite fields, given in the previous sections. Let us begin with the basic inequalities. In fact, one should be able to write most of the inequalities from subsection 5.1 in this more general setting. We give only the simplest statement of this type here:

Theorem 5.12. - Let $\left\{\zeta_{k}(s)\right\}$ be an asymptotically very exact family of zeta functions. Then

$$
w_{d_{\mathbf{e}}} \sum_{j=1}^{\infty} \lambda_{j} q^{-\frac{d_{\mathbf{e} j}}{2}} \leq \sum_{i=0}^{d_{\mathbf{e}}} \frac{\gamma_{i}}{q^{\left(d_{\mathbf{e}}-i\right) / 2}+w_{i}} .
$$

The Brauer-Siegel type results can also be proven in this setting. The following theorem includes all the function field versions of the Brauer-Siegel type results from section 3 except for the explicit ones (which can also be, in principle, established for general zeta and $L$ functions).

Theorem 5.13. - 1. For any asymptotically exact family of zeta functions $\left\{\zeta_{k}(s)\right\}$ and any $s$ with $\operatorname{Re} s>\frac{d_{\mathrm{e}}}{2}$ we have

$$
\lim _{k \rightarrow \infty} \frac{\log \zeta_{\mathbf{e}, k}(s)}{g_{k}}=\log \zeta_{\lim }(s) .
$$

If, moreover, 2 Re $s \notin \mathbb{Z}$, then

$$
\lim _{k \rightarrow \infty} \frac{\log \zeta_{k}(s)}{g_{k}}=\log \zeta_{\lim }(s) .
$$

The convergence is uniform in any domain $\frac{d_{\mathrm{e}}}{2}+\epsilon<\operatorname{Re} s<\frac{d_{\mathrm{e}}+1}{2}-\epsilon, \epsilon \in\left(0, \frac{1}{2}\right)$.

2. If $\left\{\zeta_{k}(s)\right\}$ is an asymptotically very exact family with $w_{d_{\mathbf{e}}}=1$ we have:

$$
\lim _{k \rightarrow \infty} \frac{\log \left|c_{k}\right|}{g_{k}} \leq \log \zeta_{\lim }\left(\frac{d_{\mathbf{e}}}{2}\right)
$$


where $r_{k}$ and $c_{k}$ are defined using the Taylor series expansion $\zeta_{k}(s)=c_{k}\left(s-\frac{d_{\mathbf{e}}}{2}\right)^{r_{k}}+$ $O\left(\left(s-\frac{d_{\mathbf{e}}}{2}\right)^{r_{k}+1}\right)$.

In the case of arbitrary $L$-functions the equality in (2) does not hold in general. This means that the similar questions previously discussed for function fields or elliptic curves over function fields are indeed of arithmetic nature.

Finally we will state a result on the distribution of zeroes. Let $L(s)$ be an $L$-function and let $\rho_{1}, \ldots, \rho_{g}$ be the zeroes of the corresponding polynomial $\mathcal{L}(u)$. Define $\theta_{k} \in(-\pi, \pi]$ by $\rho_{k}=q^{-d / 2} e^{i \theta_{k}}$. One can associate the measure $\Delta_{L}=\frac{1}{g} \sum_{k=1}^{g} \delta_{\theta_{k}}$ to $L(s)$.

Theorem 5.14. - Let $\left\{L_{j}(s)\right\}$ be an asymptotically very exact family of L-functions. Then the limit distribution $\lim _{j \rightarrow \infty} \Delta_{j}$ exists and has a nonnegative continuous density function given by an absolutely and uniformly convergent series $1-2 \sum_{k=1}^{\infty} \lambda_{k} \cos (k x) q^{-\frac{d k}{2}}$.

In the case of families of elliptic curves over $\mathbb{F}_{r}(t)$ P. Michel provides in [Mic] an explicit estimate for the discrepancy in the equidistribution of zeroes and a much more precise estimate for it on average.

A number of open questions concerning asymptotic properties of zeta and $L$-functions can be found in the last section of [Zyk11]. It seems that an analogue of this general asymptotic theory can be developed in the number field case (at least assuming some plausible conjectures like GRH or the Ramanujan-Peterson conjecture). This is yet to be done.

\section{References}

[BB] P. Beelen, I. Bouw. Asymptotically good towers and differential equations, Compos. Math. 141 (2005), no. 6, 1405-1424.

[BR] S. Ballet, R. Rolland. Families of curves over finite fields, PMB 2011, 5-18.

[Bra] R. Brauer. On zeta-functions of algebraic number fields, Amer. J. Math. 69, Num. 2, 1947, 243-250.

[DPZ] I. Duursma, B. Poonen, M. Zieve. Everywhere ramified towers of global function fields, Finite fields and applications, Lecture Notes in Comput. Sci., vol. 2948, Springer, Berlin, 2004, pp. 148-153. [DV] V. G. Drinfeld, S. G. Vlăduţ. The number of points of an algebraic curve (in Russian), Funktsional. Anal. i Prilozhen. 17 (1983), no. 1, 68-69.

[Elk] N. D. Elkies. Explicit towers of Drinfeld modular curves, Progress in Mathematics 202 (2001), 189-198 (Proceedings of the 3rd European Congress of Mathematics, Barcelona.

[GSR] A. Garcia, H. Stichtenoth, H.-G. Rück. On tame towers over finite fields, Journal für die Reine und Angewandte Mathematik, vol. 557(2003).

[Hin] M. Hindry. Why is it difficult to compute the Mordell-Weil group. Proceedings of the conference "Diophantine Geometry", 197-219, Ed. Scuola Normale Superiore Pisa, 2007.

[HM01] F. Hajir, C. Maire. Tamely ramified towers and discriminant bounds for number fields. Compositio Math. 128 (2001), no. 1, 35-53.

[HM02] F. Hajir, C. Maire. Tamely ramified towers and discriminant bounds for number fields. II. J. Symbolic Comput. 33 (2002), no. 4, 415-423. 
[HP] M. Hindry, A. Pacheco. An analogue of the Brauer-Siegel theorem for abelian varieties in positive characteristic, preprint.

[Iha81] Y. Ihara. Some remarks on the number of rational points of algebraic curves over finite fields, J. Fac. Sci. Univ. Tokyo Sect. IA Math., 28(3):721-724 (1982), 1981.

[Iha83] Y. Ihara. How many primes decompose completely in an infinite unramified Galois extension of a global field? J. Math. Soc. Japan 35(1983), n.4, 693-709.

[Iha06] Y. Ihara. On the Euler-Kronecker constants of global fields and primes with small norms, Algebraic geometry and number theory, 407-451, Progr. Math., 253, Birkhaüser Boston, Boston, MA, 2006.

[Iha08] Y. Ihara. On congruence monodromy problems, MSJ Memoirs 18, Math. Soc. Japan, 2008.

[IS99] H. Iwaniec, P. Sarnak. Dirichlet $L$-functions at the central point. Number theory in progress, Vol. 2 (Zakopane-Koscielisko, 1997), 941-952, de Gruyter, Berlin, 1999.

[IS00] H. Iwaniec, P. Sarnak. The nonvanishing of central values of automorphic $L$-functions and Siegel's zero, Israel J. Math. 120 (2000), 155-177.

[KT] B. E. Kunyavskii, M. A. Tsfasman. Brauer-Siegel theorem for elliptic surfaces, Int. Math. Res. Not. IMRN 2008, no. 8.

[Lab] J. Labute. Mild pro-p-groups and Galois groups of $p$-extensions of $\mathbb{Q}$, J. Reine Angew. Math., 596:155-182, 2006.

[Lan71] S. Lang. On the zeta function of number fields, Invent. Math. 12 (1971), 337-345.

[Lan94] Lang, S. Algebraic number theory (Second Edition), Graduate Texts in Mathematics 110, Springer-Verlag, New York, 1994.

[LT] G. Lachaud, M. A. Tsfasman. Formules explicites pour le nombre de points des variétés sur un corps fini, J. Reine Angew. Math. 493 (1997), 1-60.

[Leb07] P. Lebacque. Generalised Mertens and Brauer-Siegel Theorems, Acta Arith. 130 (2007), no. 4, 333-350.

[Leb09] P. Lebacque. Quelques résultats effectifs concernant les invariants de Tsfasman-Vlădut, preprint arXiv:0903.3027.

[Leb10] P. Lebacque. On Tsfasman-Vlădut invariants of infinite global fields, Int. J. Number Theory, no. 6(2010), 1419-1448.

[LZ] P. Lebacque, A. Zykin. On logarithmic derivatives of zeta functions in families of global fields, to appear at Int. J. Number Theory.

[Mic] P. Michel. Sur les zéros de fonctions $L$ sur les corps de fonctions, Math. Ann. 313 (1999), no. 2, 359-370.

[NX] H. Niederreiter, C. Xing. Rational points on curves over finite fields: theory and applications. London Mathematical Society Lecture Note Series, 285. Cambridge University Press, Cambridge, 2001.

[Odl76] A. M. Odlyzko. Lower bounds for discriminants of number fields. Acta Arith. 29(1976), 275297.

[Od190] A. M. Odlyzko. Bounds for discriminants and related estimates for class numbers, regulators and zeroes of zeta-functions: a survey of recent results. Sém. Th. Nombres Bordeaux, 1990, v.2, 119-141.

[Sch] A. Schmidt. Über pro- $p$-fundamentalgruppen markierter arithmetischer Kurven, J. Reine Angew. Math., 640:203-235, 2010.

[Ser75] J.-P. Serre. Minorations de discriminants, note of October 1975, published on pp. 240-243 in vol. 3 of Jean-Pierre Serre, Collected Papers, Springer 1986.

[Ser85] J.-P. Serre. Rational points on curves over Finite Fields, Notes of Lectures at Harvard University by F. Q. Gouvêa, 1985. 
[Sie] C. L. Siegel. Über die Classenzahl quadratischer Zahlkörper. Acta Arith. 1 (1935), 83-86.

[Sta74] H. M. Stark. Some effective cases of the Brauer-Siegel Theorem, Invent. Math. 23(1974), $135-152$.

[Tsf92] M. A. Tsfasman. Some remarks on the asymptotic number of points, Coding Theory and Algebraic Geometry, Lecture Notes in Math. 1518, 178-192, Springer-Verlag, Berlin 1992.

[Tsf95] M. A. Tsfasman. Nombre de points des surfaces sur un corps fini, Algebraic Geometry and Coding Theory, Proceedings AGCT-4, De Gruyter, 1995.

[TV92] M. A. Tsfasman, S. G. Vlăduţ. Algebraic-geometric codes. Translated from the Russian by the authors. Mathematics and its Applications (Soviet Series), 58. Kluwer Academic Publishers Group, Dordrecht, 1991.

[TV97] M. A. Tsfasman, S. G. Vlăduţ. Asymptotic properties of zeta-functions, J. Math. Sci. 84 (1997), Num. 5, 1445-1467.

[TV02] M. A. Tsfasman, S. G. Vlăduţ. Inifinite global fields and the generalized Brauer-Siegel Theorem, Moscow Mathematical Journal, Vol. 2 (2002), Num. 2, 329-402.

[TVN] M. A. Tsfasman, S. G. Vlădut, D. Nogin. Algebraic geometric codes: basic notions, Mathematical Surveys and Monographs, 139, American Mathematical Society, Providence, RI, 2007.

[TVZ] M. A. Tsfasman, S. G. Vlăduţ, Th. Zink. Modular curves, Shimura curves, and Goppa codes, better than Varshamov-Gilbert bound, Math. Nachr. 109(1982), 21-28.

[Ulm02] D. Ulmer. Elliptic curves with high rank over function fields, Annals of Math. 155(2002), $295-315$.

[Zim] R. Zimmert. Ideale kleiner Norm in Idealklassen und eine Regulatorabschatzung, Invent. Math. 62(1981), 367-380.

[Zyk05] A. Zykin. Brauer-Siegel and Tsfasman-Vlădut theorems for almost normal extensions of global fields, Moscow Mathematical Journal, Vol. 5 (2005), Num 4, 961-968.

[Zyk09] A. Zykin. On the generalizations of the Brauer-Siegel theorem. Proceedings of the Conference AGCT 11 (2007), Contemp. Math. series, AMS, 2009.

[Zy10] A. Zykin. Asymptotic properties of Dedekind zeta functions in families of number fields, Journal de Théorie des Nombres de Bordeaux 22(2010), no. 3, 689-696.

[Zyk11] A. Zykin. Asymptotic properties of zeta functions over finite fields, preprint.

02 mai 2011

Philippe Lebacque, Laboratoire de Mathématiques de Besançon, UFR Sciences et techniques, 16, route de Gray 25030 Besançon, France • E-mail : philippe.lebacque@univ-fcomte.fr

Alexey Zykin, State University - Higher School of Economics, Department of Mathematics, Laboratory of Algebraic Geometry, SU-HSE, 117312, 7 Vavilova Str., Moscow, Russia

Institute for Information Transmission Problems of the Russian Academy of Sciences, Laboratoire

Poncelet (UMI 2615) • E-mail : alzykin@gmail.com 